\title{
EVALUACIÓN HIGIÉNICA DE RIESGOS BIOLÓGICOS DEL TRABAJO EN ESTABULARIO DE UN CENTRO DE INVESTIGACIÓN SANITARIA
}

\author{
BIOLOGICAL RISK ASSESSMENT IN ANIMAL FACILITIES OF A SANITARY \\ INVESTIGATION CENTRE
}

\author{
CAROLINA MIRAZ NOVÁS(1)
}

(1) Complejo Hospitalario Universitario. A Coruña. España.

\section{RESUMEN}

Objetivo: El propósito del presente trabajo es evaluar los riesgos biológicos a los que están expuestos los trabajadores del estabulario de la Unidad de Investigación Sanitaria con el fin de determinar la necesidad de adoptar medidas preventivas que permitan controlar el riesgo, determinar dichas medidas y conocer la aplicabilidad de la metodología elegida en el caso específico del trabajo con animales.

Metodología: Se utilizó la metodología de evaluación BIOGAVAL propuesta por el Gabinete de Seguridad e Higiene en el Trabajo de Valencia para la evaluación del riesgo biológico existente en los puestos de técnico de animalario y veterinario. El método seleccionado requiere, para la determinación del nivel de riesgo, la identificación de los microorganismos involucrados, el daño a la salud, las vías de transmisión, la posibilidad de vacunación y el porcentaje de personal vacunado o las medidas higiénicas existentes en el momento de llevar a cabo la evaluación.

Resultados: Los resultados de la evaluación de los puestos de trabajo indican la necesidad de medidas preventivas, en especial encaminadas al control de Leptospira y Brucella. Una vez adoptadas las medidas de prevención se observa que el nivel de riesgo de todos los agentes se sitúa en valores inferiores a los límites de exposición biológica establecidos por el método. Se constata la falta de datos determinantes para la valoración numérica del riesgo relativo a algunos agentes identificados. Se considera que los niveles de riesgo emanados del método pueden ser superiores a los reales al no contabilizarse en el "Formulario de medidas higiénicas adoptadas" aquellas medidas realizadas sobre los propios animales.

Conclusiones: La metodología de evaluación prueba ser un método ágil, ordenado y relativamente sencillo para la determinación del riesgo biológico al que están expuestos los trabajadores. El método de evaluación BIOGAVAL supone una herramienta muy práctica a la hora de sistematizar evaluaciones de elevada complejidad dada por su característica de indeterminación de presencia de los agentes biológicos, si bien su aplicación se ve drásticamente mermada debido a la ausencia de datos estadísticos fiables sobre variables determinantes como duración de incapacidades temporales o tasas de incidencias para muchos agentes biológicos. El método proporciona orientación sobre el tipo de medidas preventivas a adoptar. Por otro lado se propone una ampliación en el "Formulario de Medidas Higiénicas Adoptadas" con la intención de recoger el hecho característico del trabajo con animales.

Palabras clave: Riesgos laborales, higiene industrial, exposición a agentes biológicos.

\section{ABSTRACT}

Aims: The purpose of this study is to assess the biological risks to which workers in the animal facilities of the Health Research Unit are exposed in order to determine the need for preventive measures to control risk, determine those measures and test methodology chosen in the specific case of working with animals.

Methods: BIOGAVAL evaluation methodology proposed by Office of Security and Hygiene in the Work of Valencia was used to evaluate the technician and veterinarian biological risks. The chosen methodology needs, for the determination of the risk level, identification of the involved microorganisms, damage to the health, routes of transmission, possibility of vaccination and percentage of vaccinated personnel and the hygienic existing measures in the moment to carry out the evaluation.

Results: The results show the need of preventive measures, especially directed to the control of Leptospira and Brucella. Once adopted the measures, the risk level for all agents places in values lower than the level established by the method. It is stated the lack of determinant information for the numerical risk evaluation relative to some identified agents. The levels of risk came from the method could be superior to the real ones as in the "Form of hygienic adopted measures" there're not considered those measures realized on the proper animals.

Conclusions: The methodology of evaluation proves to be an agile, orderly and relatively simple method for the determination of the biological risk to which the workers are exposed. The method of evaluation BIOGAVAL is a practical tool as systematized evaluations of high complexity, due to its characteristic uncertainty on the presence of the biological agents, though its application results drastically reduced due to the absence of statistical trustworthy information on determinant variables as duration of temporary disabilities or rates of incidents for some biological agents. The method provides orientation on the type of preventive measures to adopt. It is proposed an extension in the "Form of Hygienic Adopted Measures" to take into account the fact of the working with animals.

Key words: Occupational risks, occupational hygiene, exposure to biological agents.

\section{Correspondencia:}

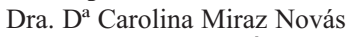

T. Hixiene Industrial Área A Coruña

C.H.U. A Coruña

As Xubias de Arriba, 84

15006 A Coruña. España

Tel. 981178000 (ext. 295102) 


\section{INTRODUCCIÓN}

La protección eficaz de la salud de los trabajadores pasa por un conocimiento exhaustivo de los riesgos a los que están expuestos.

A este conocimiento se llega a través de evaluaciones de mayor o menor complejidad, que en el caso específico de los agentes biológicos (AB) suelen conllevar un elevado grado de especialización, tal y como se plasma en los requisitos establecidos por la Guía Técnica para la evaluación y prevención de los riesgos relacionados con la exposición a $A B$ publicada por el Instituto Nacional de Seguridad e Higiene en el Trabajo (INSHT) ${ }^{i}$ para la aplicación del R.D. 664/972.

Ahondando en esta complejidad, el propio INSHT indica en la Guía Técnica ${ }^{1}$ que la evaluación de riesgos en actividades en las que los $\mathrm{AB}$ se presentan de forma incidental es más compleja que una evaluación en una actividad de manipulación deliberada, debido esencialmente a la incertidumbre de presencia del agente y la dificultad que esto asocia a su determinación, unido a la ausencia de valores límite de exposición o a lo desaconsejado del muestreo y análisis sistemáticos, como se realizaría en caso de agentes químicos.

Con el trabajo presentado en este artículo se pretende profundizar en el conocimiento del riesgo asociado a los $\mathrm{AB}$ al que está expuesto el personal del estabulario de la Unidad de Investigación Sanitaria en el que no existe manipulación deliberada de $\mathrm{AB}$, establecer medidas preventivas que permitan minimizar el riesgo y conocer la aplicabilidad de la metodología BIOGAVAL ${ }^{3}$ propuesta por el Gabinete de Seguridad e Higiene en el Trabajo de Valencia en puestos de trabajo asociados al contacto con animales.

\section{MATERIAL Y MÉTODOS}

El método utilizado en la evaluación es el BIOGAVAL $^{3}$ anteriormente citado y diseñado explícitamente para realizar evaluaciones de riesgos biológicos en actividades en las que no exista una intención deliberada de trabajar con dichos agentes. Consecuentemente se han seguido las etapas propuestas por la metodología:

\subsection{Determinación de los puestos a evaluar}

Tal y como establece la legislación de aplicación y recoge la metodología, la evaluación de riesgos se realiza por puesto y se consideran incluidos en el mismo puesto aquellos trabajadores cuya asignación de tareas y entorno de trabajo, determinen un elevado porcentaje de homogeneidad en riesgos, grados de exposición y gravedad de las consecuencias.

En base a estas consideraciones los trabajadores del estabulario se han agrupado en dos puestos de trabajo: técnico de animalario y veterinario.

Los puestos de trabajo sometidos a evaluación se desarrollan en el área de estabulario perteneciente a una unidad de investigación sanitaria.

En el estabulario se realizan actividades de cría, cuarentena, mantenimiento y estudio de diferentes animales, en su mayoría ratas, ratones, conejos, ovejas y cerdos.

Las tareas realizadas por los técnicos de animalario son:

- Limpieza de instalaciones, jaulas, etc.

- Alimentación de animales

- Preparación de animales para intervenciones: manipulaciones, tratamientos y extracciones de sangre

- Eliminación de cadáveres

Por su parte, las actividades realizadas por los veterinarios son:

- Administración de tratamientos

- Cuidados postoperatorios

- Anestesias e intervenciones quirúrgicas (desarrolladas en el área de cirugía experimental)

\subsection{Identificación del agente microbiológico implicado}

La característica diferenciadora de los trabajos que no tienen por objeto la manipulación deliberada de $\mathrm{AB}$ es la gran diversidad de agentes que con mayor o menor probabilidad pueden asociarse a los mismos. En base a la información científica disponible $^{4,5}$, el estudio de los puestos de trabajo y los datos aportados por el personal, se identificaron los siguientes $\mathrm{AB}$ como posibles riesgos en los puestos de trabajo en estudio. 
Tabla I, Agentes biológicos identificados

\begin{tabular}{l|l|l}
\hline Enfermidade & Axente biolóxico & Grupo \\
\hline Brucelosis & Brucella spp & 3 \\
\hline Tuberculosis & Mycobacterium tuberculosis M. bovis & 3 \\
\hline Leptospirosis & Leptospira spp & 2 \\
\hline Salmonelosis & Salmonella spp & 3 \\
\hline Campilobacteriosis & Campylobacter fetus jejuni & 2 \\
\hline Dermatomicosis: & Microsporum & $2 \mathrm{~A}$ \\
Tinea capitis & Tricophyton & 2 \\
Tinea glabrosa & Epidermophyton & $2 \mathrm{~A}$ \\
\hline Fiebre por mordedura de rata & Streptobacillus moniliformis & 2 \\
\hline Ameniasis & Entamoeba histolytica & 2 \\
\hline Disentería bacilar & Shigella dysenteriae & $2 / 3 \mathrm{~T}$ \\
\hline Melioidosis & Burkholderia pseudomallei & 3 \\
\hline
\end{tabular}

\subsection{Cuantificación de las variables determinan- tes del riesgo}

Para la cuantificación del riesgo se tiene en cuenta:

- El daño a la salud que puede producir el microorganismo (días de baja que podría suponer padecer la enfermedad ${ }^{6}$, posibles secuelas o fallecimiento del trabajador)

- Vías de transmisión del agente (directa, indirecta o aérea)

- Tasa de incidencia durante el año anterior de la enfermedad en la población

- Existencia o no de una vacuna y el porcentaje de trabajadores vacunados

- Frecuencia de realización de las pareas en las que existe el riesgo en proceso de evaluación

- Medidas higiénicas adoptadas en el lugar de trabajo, que podrán suponer una disminución en el peso de las vías de transmisión y el daño producido.

La fórmula de cálculo del nivel de riesgo es:

$$
R=\left(D^{\prime} V\right)+T^{\prime}+I+F
$$

Donde:

$\mathrm{R}=$ nivel de riesgo

$\mathrm{D}^{\prime}=$ daño (corregido por las medidas higiénicas existentes)

$\mathrm{V}=$ valor asociado por el método según el porcentaje de personal vacunado

$\mathrm{T}^{\prime}=$ vía de transmisión (corregido por las medidas higiénicas)

$\mathrm{I}=$ tasa de incidencia en el año anterior

$\mathrm{F}=$ frecuencia de exposición a las tareas de riesgo

\section{RESULTADOS}

En la búsqueda de los datos necesario para el cálculo de los niveles de riesgo se comprobó la escasez de fuentes fiables en las que basar la obtención de las variables determinantes del riesgo, como es el caso de la duración de incapacidades temporales o tasas de incidencia en el año anterior para algunos $\mathrm{AB}$.

Para los casos de aquellos agentes en los cuales se ha tenido acceso a todos los datos necesarios, los resultados obtenidos se muestran en las figuras 1 y 2 para los puestos de técnico de animalario y veterinario respectivamente. 
Figura 1. Niveles de riesgo biológico para el puesto de técnico de animalario.

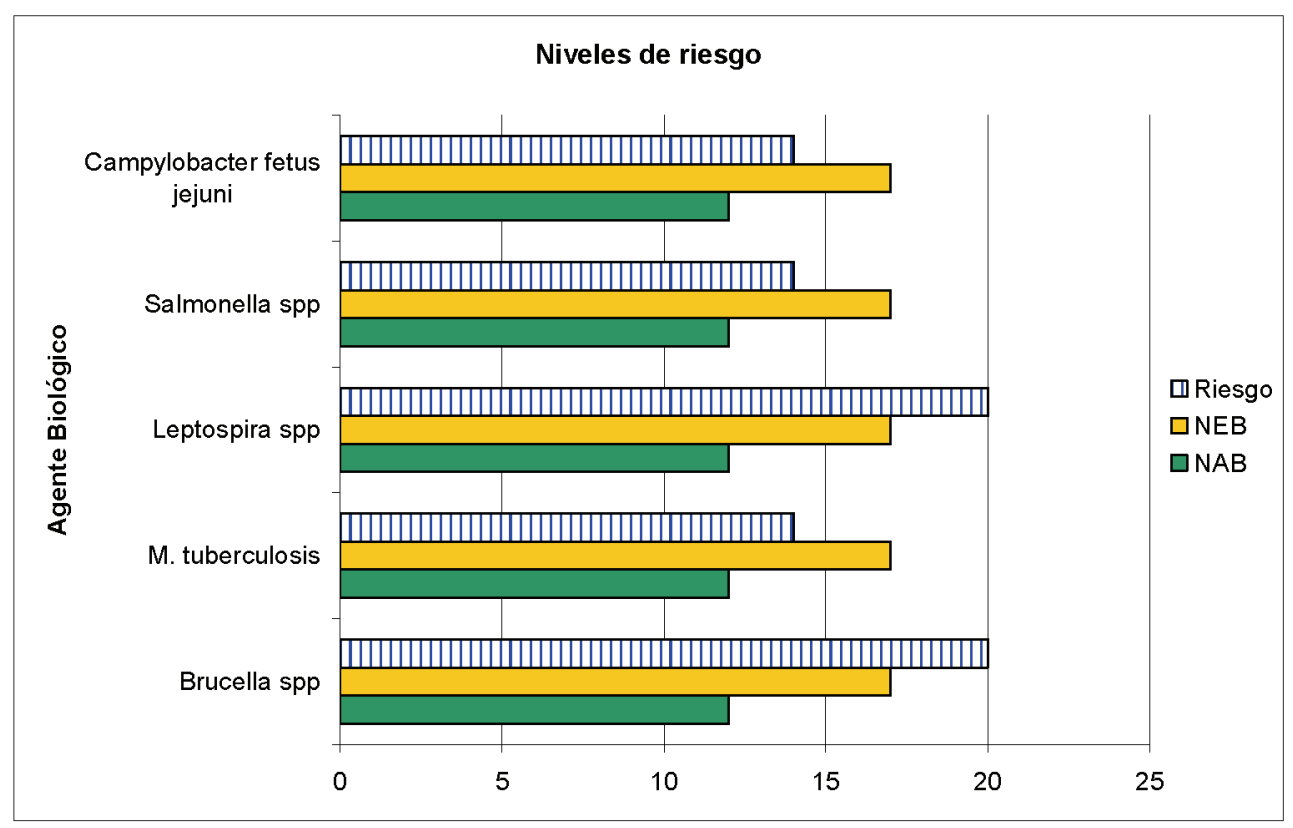

Figura 2. Niveles de riesgo biológico para el puesto de veterinario.

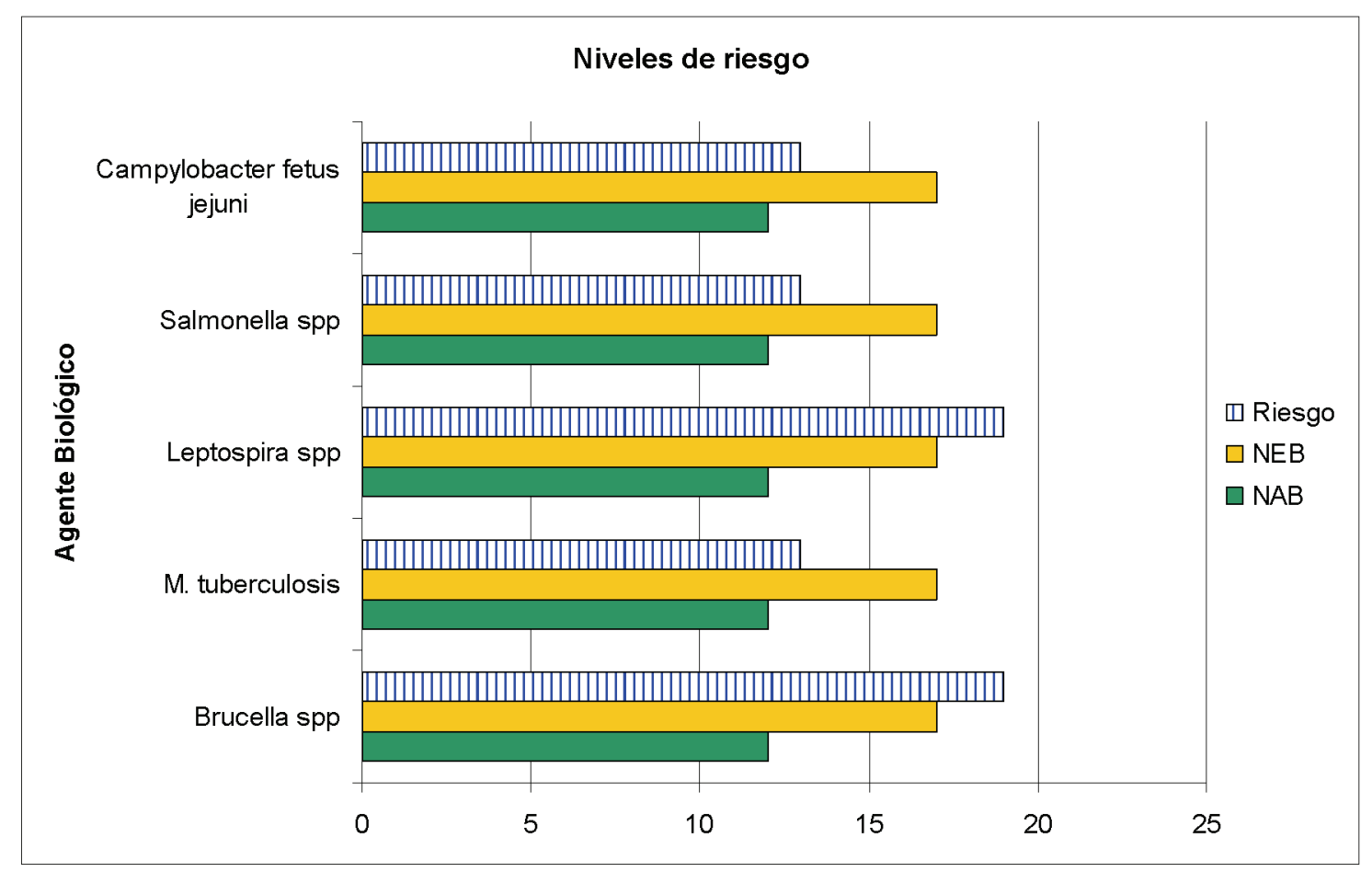


En ambas figuras los niveles de riesgo obtenidos aparecen representados junto a los valores de referencia planteados por el método:

- Nivel de acción biológica (NAB): valor a partir del cual deberán tomarse medidas de tipo preventivo para intentar disminuir la exposición.

- Límite de exposición biológica (LEB): valor que en ningún caso debe superarse.
Para los casos en los que no se han encontrados datos para el cálculo de los índices se optó por el establecimiento de medidas preventivas basadas en datos procedentes de las "Fichas de Datos de Seguridad para Sustancias Infecciosas" $" 7,8$.

Como se observa en las figuras 1 y 2 , para ambos puestos se supera el límite en los casos de Leptóspira y Brucella, por lo que se propusieron una serie de medidas preventivas tras la aplicación de las cuales los niveles de riesgo disminuyen para todos los agentes, como se observa en las figuras 3 y 4 .

Figura 3, Niveles de riesgo biológico para el puesto de técnico de animalario, tras aplicación de medidas preventivas.

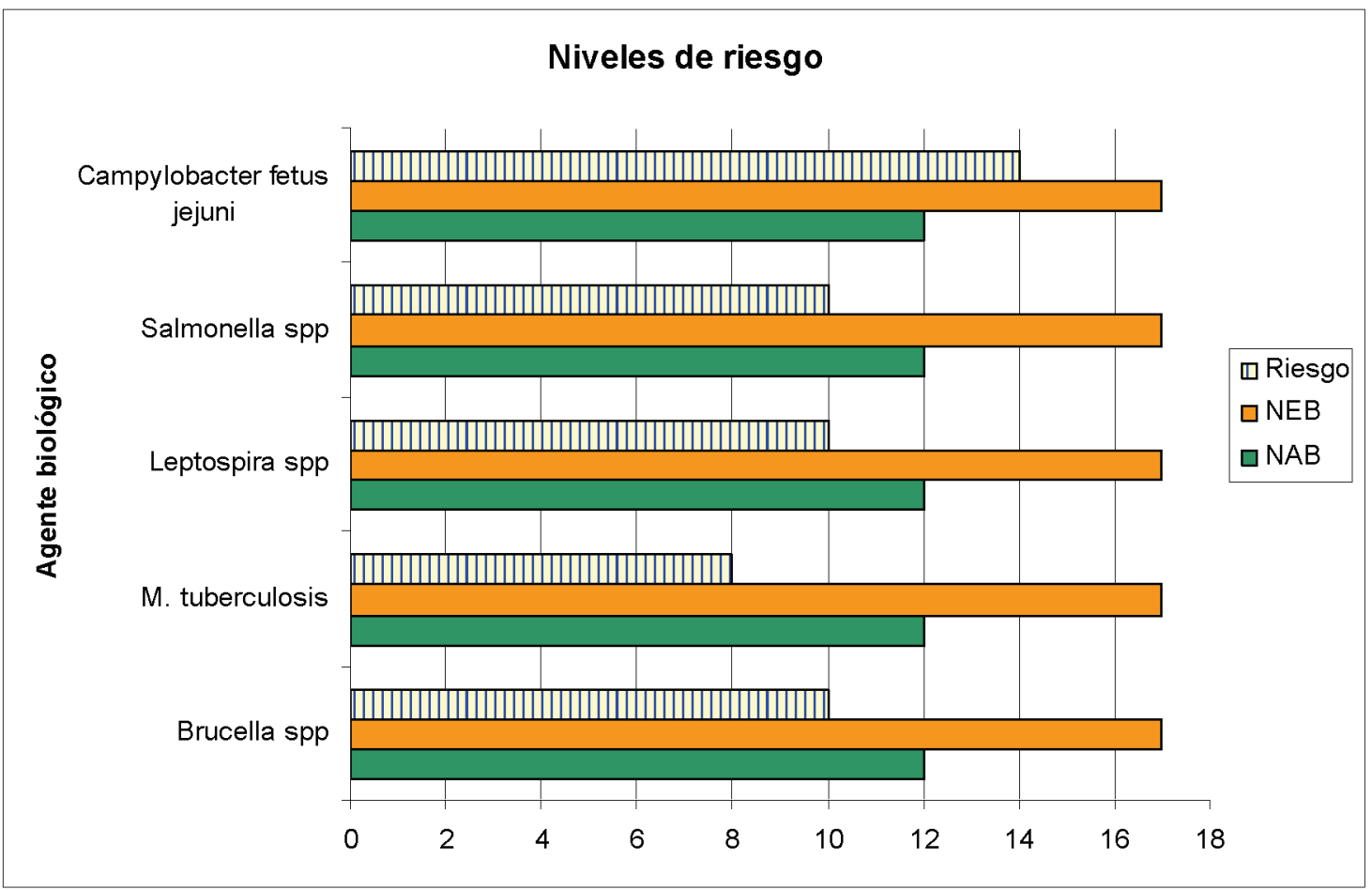
son:

Algunas de las medidas preventivas propuestas

- Actualización de los calendarios de vacunación

- Información y formación a los trabajadores en forma de instrucciones de trabajo

- Controlar el mantenimiento de las condiciones de uso de los equipos de protección personal

- Dotación de taquillas dobles

- Dotación de sistema de lavaojos de emergencia

\section{CONCLUSIONES}

El método resulta una forma ágil de evaluar la exposición a riesgo biológico de forma muy específica aunque su aplicación depende de datos de los que no se dispone para todos los agentes.

Proporciona información precisa sobre las líneas a seguir en las medidas preventivas necesarias para la contención del riesgo, a través del "Formulario de medidas higiénicas adoptadas". 
Figura 4, Niveles de riesgo biológico para el puesto de veterinario, tras aplicación de medidas preventivas.

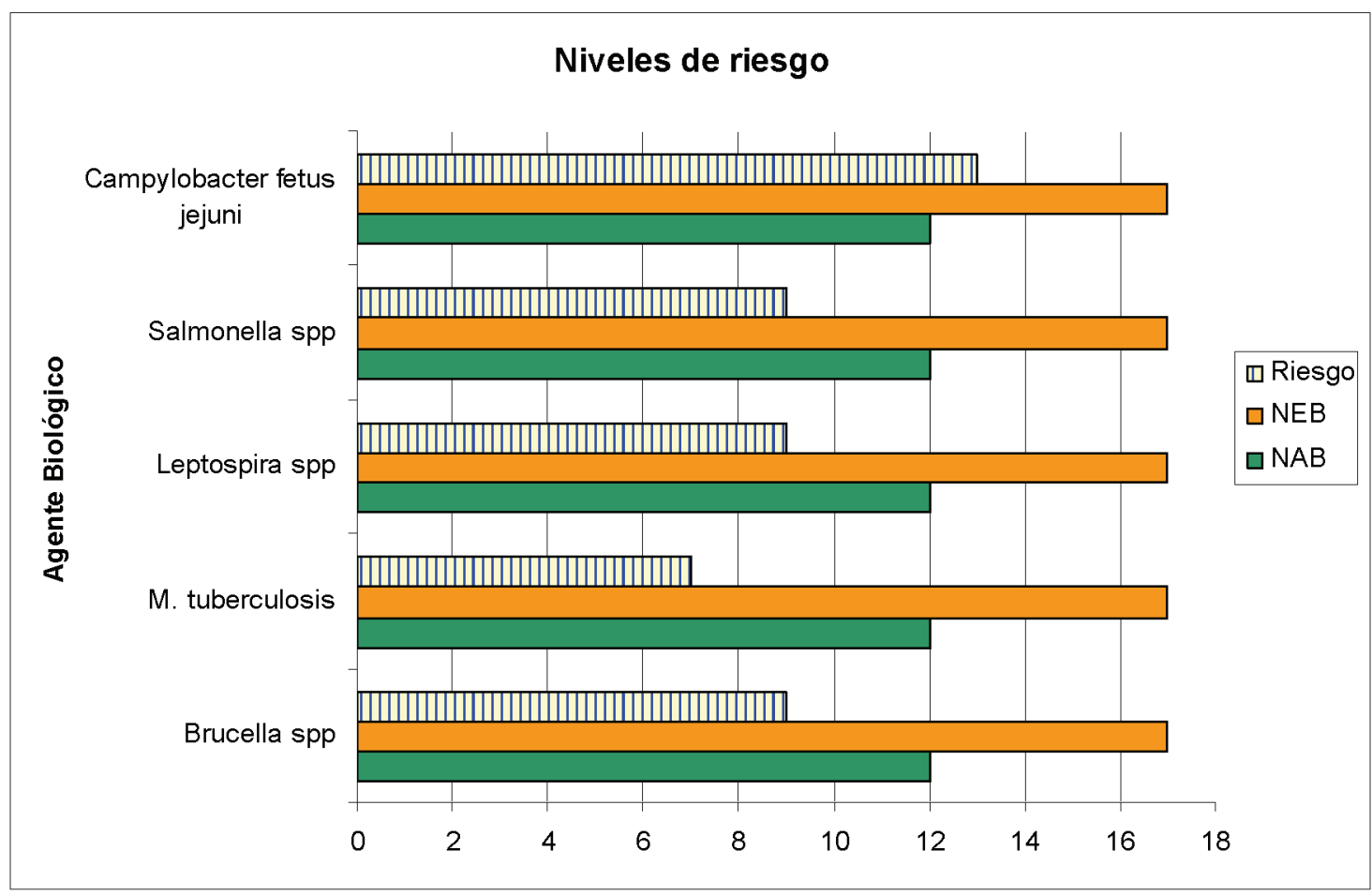

Una vez adoptadas las medidas higiénicas propuestas los valores de riesgo se encuentran en valores aceptables en todos los casos, aunque condicionantes como la inexistencia de vacuna eficaz elevan el riesgo considerablemente y dificultan la minimización del nivel de riesgo, como es el caso del Campilobacter fetus jejuni.

Para el caso concreto del trabajo con animales de experimentación, se observó la inexistencia en el "Formulario de medidas higiénicas adoptadas", incluido en el método de evaluación, de referencias a los controles veterinarios a los que son sometidos todos los animales antes de ser aceptados en las unidad de investigación que incluyen test de tuberculosis, salmonella, etc y que contribuyen en gran medida a controlar el riesgo biológico por lo que los niveles de riesgo obtenidos en evaluación serían superiores a los reales. Esta observación fue puesta en conocimiento del coordinador y director del manual de evaluación BIOGAVAL ${ }^{3}$.

Se propuso incluir una pregunta que contemplase la realización de controles veterinarios a los animales y en función de aquellos agentes biológicos que estén controlados se produciría para caso concreto una disminución en la transmisión del mismo.

En lo referente a los $\mathrm{AB}$ para los que no se está disposición de todos los datos necesarios para la obtención del nivel de riesgo biológico, cabe destacar que los datos obtenidos en la aplicación del método: identificación, posibilidad de vacunación, vías de transmisión y frecuencia de exposición, si bien no son suficientes para la obtención del valor numérico, si ofrecen información que permite establecer medidas preventivas y controles que mejoren las condiciones de seguridad y salud 


\section{$\overline{\text { BIBLIOGRAFÍA }}$}

1. Ministerio de Trabajo y Asuntos sociales. Instituto Nacional de Seguridad e higiene en el Trabajo. Guía técnica para la evaluación y prevención de los riesgos relacionados con la exposición a agentes biológicos. 1998 .

2. R.D. 664/97, de 12 de mayo, sobre la protección de los trabajadores contra los riesgos relacionados con la exposición a agentes biológicos durante el trabajo.

3. Llorca Rubio JL. Manual práctico para la evaluación del riesgo biológico en actividades laborales diversas (BIOGAVAL). Valencia. Generalitat Valenciana. 2004.

4. Organiazación Mundial para la salud animal. Disponible en la World Wide Web: <http://www. oie.int/eng/en_index.htm>
5. Zúñiga Jesús M, Tur Marí J, Micolo S N. Ciencia y tecnología en protección y experimentación animal. McGrawHill. Iberoamericana.

6. INSALUD. Subdirección General de Coordinación Administrativa. Manual de Gestión de la incapacidad temporal. Madrid. INSALUD. 1999

7. Public Health Agency of Canada. Fichas de Datos de Seguridad para Sustancias Infecciosas. Disponibles en la World Wide Web: <http://www. phac- aspc.gc.ca/mods-ftss/index.html\#1>

8. Center for Disease Control and Prevention. Disponible en la World Wide Web: <http://www. cdc.gov/ncidod/dbmd/diseaseinfo 